\title{
Macrofauna bentónica de las playas de arena del Área de Conservación Osa, Puntarenas, Pacífico Sur de Costa Rica
}

\author{
Marco Corrales-Ugalde ${ }^{1,3}$ \& Jeffrey A. Sibaja-Cordero ${ }^{2,3}$ \\ 1. Consultor independiente para Fundación Keto; mcugalde88@gmail.com \\ 2. Museo de Zoología, Escuela de Biología, Universidad de Costa Rica, San Pedro, San José, Costa Rica, 11501-2060. \\ 3. Centro de Investigación en Ciencias del Mar y Limnología (CIMAR), Ciudad de la Investigación, Universidad de \\ Costa Rica, Costa Rica.
}

Recibido 11-VIII-2014. Corregido 20-XI-2014. Aceptado 22-XII-2014.

\begin{abstract}
Benthic macrofauna found in Sandy beaches from Osa Conservation Area, Puntarenas, Southern Pacific coast of Costa Rica. The diversity and species composition of the intertidal sandy beaches in the southern Pacific coast of Costa Rica were studied by means of cores collected along perpendicular transects to the beaches. The numbers of strictly marine species varied between 5 to 13 taxa, representing an intermediate value compared to those previously reported for these environments in Costa Rica. The isopod Cirolana salvadorensis was the dominant species in the supralittoral zone, whereas polychaete worms belonging to the families Nereididae and Pisionidae dominated the low intertidal zone. Others organisms collected in the beaches were the crabs of the genus Uca, anomurans crabs (Emerita), sand dollars (Mellita longifissa) and several taxa of polychaete tubeworms, such as the Onuphidae, Spionidae, Magelonidae, and Glyceridae. The high faunal difference among the sites is possibly explained by their exposure to the wave energy, with fewer individuals in the more reflective beaches. In addition, human activities might also be responsible for the low infaunal diversity found in some of these beaches. This is the first effort to describe the benthonic fauna of beaches from this area. Rev. Biol. Trop. 63 (Suppl. 1): 273-285. Epub 2015 April 01.
\end{abstract}

Key words: Sand beach, vertical zonation, marine biodiversity, Punta Burica, human impacts.

Las playas de arena han sido bien estudiadas en regiones de clima templado, de donde se conoce los principales factores que influyen en la distribución y la variabilidad de su fauna, como lo son el sedimento, el oleaje, y el tiempo de desecación en marea baja (Little, 2000). Otros ambientes como playones fangosos han sido estudiados y se ha encontrado patrones de zonación relacionados con el nivel de la marea (Dittmann, 2000). También, se ha estudiado como la morfología de las playas puede hacer variar dichos patrones (Brazeiro, Rozbaczylo \& Fariña, 1998).

Los estudios en América Central sobre zonas de entre mareas son pocos, principalmente realizados en El Salvador, Panamá y Costa Rica (Alongi, 1990; Dittmann \& Vargas, 2001). Para Costa Rica, en lo referente a sustratos blandos, la mayoría de los estudios de macrofauna y meiofauna son de playas fangosas del Golfo de Nicoya, como Punta Morales (De la Cruz \& Vargas, 1987; Vargas, 1987, 1988a, 1988b; Dittmann \& Vargas, 2001). Las playas de arena de la costa pacífica han sido estudiadas por Dexter (1974) con tres playas del Pacífico Norte, tres del Golfo de Nicoya y dos en el Pacífico Central, para las cuales reporta distribución y densidad de 52 especies.

Las regiones del Pacífico Sur de Costa Rica han sido poco estudiadas ecológicamente. Quesada-Alpízar y Cortés (2006) realizan una revisión de la literatura existente sobre el ambiente marino del Pacífico Sur de Costa Rica, donde indican que la mayor cantidad de información existente son registros de colectas taxonómicas de grupos de moluscos y anélidos 
de varias playas en Golfo Dulce (Høisæter, 1998; Dean, 2004), además indican que la biodiversidad de sustratos suaves es un aspecto de los ecosistemas marinos que no se ha estudiado en la región. Cortés y Jiménez (1996), reportan poblaciones de Coenobita compressus y Uca spp. en las playas arenosas de la península de Osa. La región de Punta Burica, más al sur de Golfo Dulce, no cuenta con estudios sobre la composición de la fauna de entre mareas, y al ser una región poco habitada cuenta con un área marino costera poco alterada o intacta en la mayor parte de la región. Lo mismo sucede con el litoral de la región de la desembocadura del sistema estuarino Térraba-Sierpe y las playas aledañas al Parque Nacional MarinoBallena, regiones las cuales son visitadas tanto por turistas como pescadores artesanales que extraen ciertos recursos alimenticios en las zonas de entre mareas. El presente trabajo busca describir de manera preliminar, dicha fauna y sus patrones de distribución en las playas del Pacífico Sur de Costa Rica, relacionando estos patrones con parámetros como la zonación y la exposición al oleaje (McLachlan \& Jaramillo, 1995; Little, 2000) y el posible impacto antropogénico.

\section{MATERIALES Y MÉTODOS}

Descripción de la zona de estudio: Las zonas entre mareas de las playas arenosas del Área de Conservación Osa presentan diferencias en la inclinación respecto a la acción directa del oleaje o en el tiempo de exposición durante la marea baja. Las playas de tipo disipativas se generan donde la exposición al oleaje es considerable; se les llama disipativas porque la energía se disipa en zonas de rompiente ancha, lo cual produce poca pendiente $\mathrm{y}$ permite que se acumulen sedimentos con tamaños de grano pequeños $(<200 \mu \mathrm{m})$ (Little, 2000). Aquellas playas de rompiente estrecha presentan pendientes marcadas, y se les denomina reflectivas. En estas playas el sedimento presenta tamaños de grano mayores que en las playas disipativas, y puede incluir grava fina y piedras (>4mm, Gray \& Elliot, 2009) (Little,
2000). En el informe GRUAS II (2009), se definen cuatro Unidades Ecológicas Marinas (UEM), para el Pacífico Sur de nuestro país, las cuales fueron analizadas en este estudio: Ballena, Térraba, Corcovado y Burica.

UEM Ballena, Térraba y Corcovado: Las Playas Dominical, Dominicalito, Punta Uvita, Ballena, Piñuela y Ventanas se caracterizan por ser de tipo disipativas, son bastante amplias y presentan poca inclinación. Las playas Garza y Palo Seco que se encuentran en la desembocadura del Térraba- Sierpe son similares a las anteriores, sin embargo playa Violines presenta sedimento más fino y menos inclinación que las anteriores. En la península de Osa, se estudiaron las playas San Josecito, San Pedrillo y La Chancha. Estas playas son de tipo reflectivas, presentan mayor inclinación y arena más gruesa.

UEM Burica: En la región de Punta Burica, Punta Banco presenta playas de arena de extensión amplia y poca inclinación (Fig. 1), del tipo disipativas. Hacia el sur se encuentra de nuevo una playa de arena con cimas y valles de guijarros hasta la región de Caña Blanca, de pendiente marcada, del tipo reflectiva. En medio de éstas se ubica playa Coco, más bien intermedia entre reflectiva y disipativa. Las playas después de este punto desaparecen al contar la costa con una pared de acantilados, donde aparecen de nuevo pequeñas playas en La Palma y en la desembocadura del río Peñitas, las cuales son de pendiente pronunciada, gravosas y reflectivas (Fig. 1). Desde ahí hasta el río Peña hay playas de arena separadas por formaciones rocosas de pendiente moderada, algo reflectivas (Barco, Peña). Las zonas de arena se hacen extensas en amplitud y de escasa inclinación desde el río Peña hasta Punta Mangle (Fig. 1). Son más amplias las playas de Venegas, Chomba y Mangle (disipativas), que Palito o Clarita, donde se estrecha la franja de arena y son intermedias.

Metodología: Para la recolección de las muestras de arena se utilizaron barrenos de 


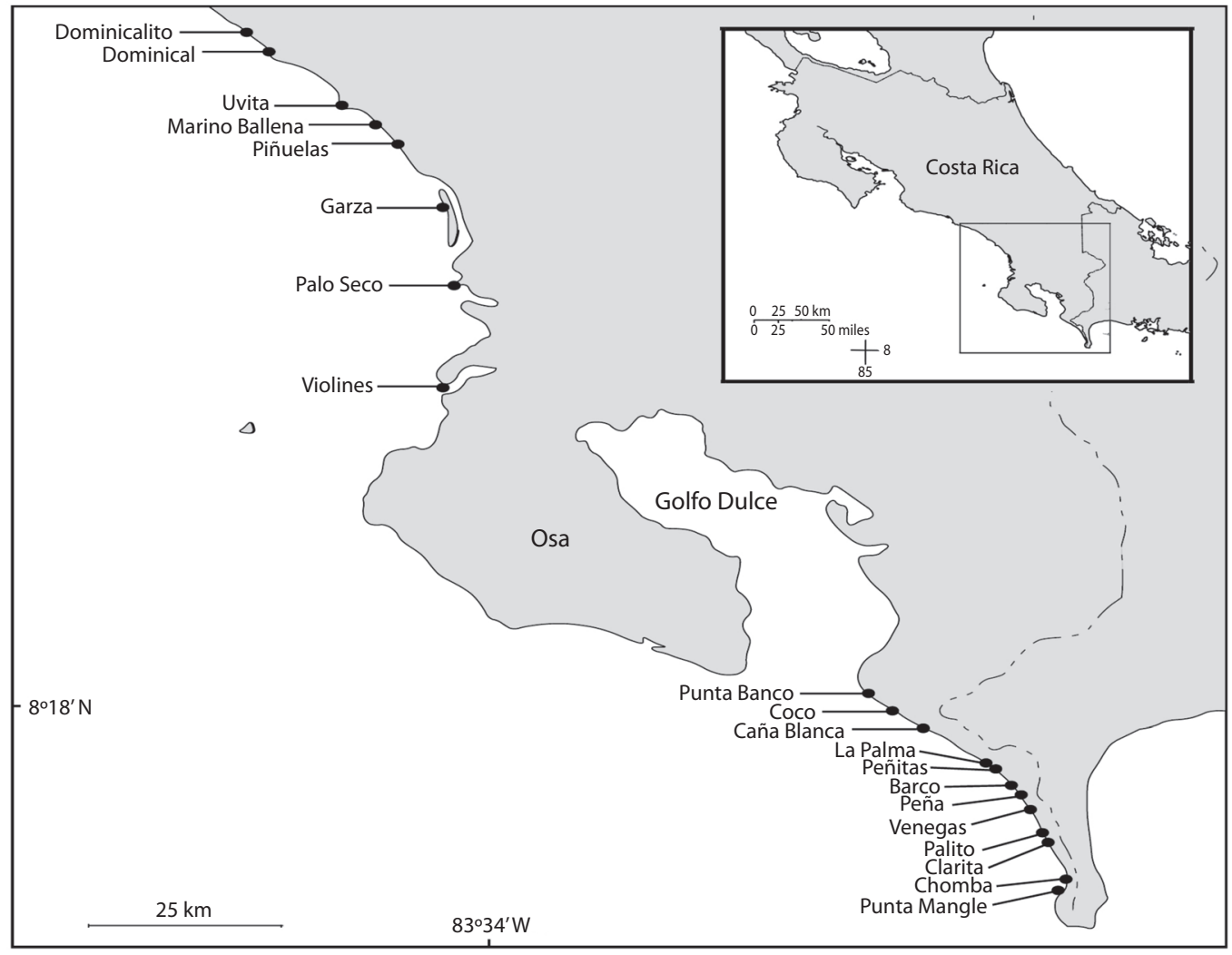

Fig. 1. Ubicación de las playas de arena donde se recolectaron barrenos. Puntarenas, Costa Rica. Enero 2008 y abril 2012. Fig. 1. Location of the sampled sandy beaches. Puntarenas, Costa Rica. January 2008 and April 2012.

18.86 y de $15.9 \mathrm{~cm}^{2}$, introducidos a $15 \mathrm{~cm}$ de profundidad en el sedimento (Vargas, 1987). Se evaluaron 26 transectos perpendiculares a la playa, para un total de 103 barrenos. Se tomó una muestra en cada uno de los estratos de entre mareas, tras dividir la franja expuesta de manera equidistante según Dexter (1974). Cada muestra se tamizó para retener los organismos pertenecientes a la macrofauna $(\geq 500 \mu \mathrm{m})$ (Dexter, 1974; Vargas, 1987). Las muestras provenientes de la UEM Burica se tamizaron con una malla de $500 \mu \mathrm{m}$, mientras que las muestras de las UEM Ballena y Térraba fueron tamizadas con una malla de $300 \mu \mathrm{m}$. Las muestras se analizaron y contabilizó el número de individuos en laboratorio con el estereoscopio. Durante el análisis de las muestras provenientes de Ballena y Térraba, se excluyó a los organismos considerados como meiofauna por Giere (2008).

La estructura del sedimento de playas estudiadas de la UEM Corcovado, compuestas principalmente de arena gruesa, gránulos y guijarros, dificultó la recolecta de los barrenos por lo cual dichas muestras no se incluyeron en este estudio. En estas playas se realizaron recolectas cualitativas de sedimento, los cuales fueron tamizados con una malla de $300 \mu \mathrm{m}$ para retener a los organismos los cuales fueron identificados en el laboratorio al taxón más específico posible.

En el playón arenoso de Playa Barco, se realizó una exploración removiendo el sedimento con una pala desde el supralitoral hasta el infralitoral y se recolectaron los organismos apreciables a simple vista para su posterior 
identificación y caracterizar cualitativamente el patrón de distribución. Además, se puso atención en los impactos humanos que se dan en las diferentes zonas del área de estudio y que pueden afectar la fauna de las playas de arena.

Análisis de datos: Para caracterizar la fauna se calculó la densidad por metro cuadrado para la zona en general, además de los índices de Shannon Weiner y la equidad. La diferencias en riqueza de taxones y abundancia total se evaluó con pruebas de chi cuadrado $\left(\chi^{2}\right)$. Se utilizó un análisis de conglomerados con distancia euclidiana por el método de unión simple de datos de abundancia de las especies en cada localidad, transformados por Log $(\mathrm{x}+1)$, para ver la similitud en la composición de la infauna entre sitios, y según si la playa era disipativa, intermedia o reflectiva (Hammer, Harper \& Ryan, 2001).

\section{RESULTADOS}

La fauna de sustratos blandos estrictamente marina fue de 12 especies en Punta Burica, 11 en Marino-Ballena, 13 especies para SierpeTérraba y 5 para Corcovado. En general en los 103 barrenos, se encontraron siete familias de gusanos poliquetos (Cuadro 1). Fueron menos frecuentes los habitantes de galerías y túneles como Spionidae y Glyceridae. La familia Onuphidae sólo se encontró en playa Violines. Dentro de los artrópodos llama la atención la presencia de hexápodos como colémbolos, coleópteros y dípteros, aunque con presencia y abundancia baja o rara. Entre los crustáceos se encontró el isópodo Cirolana salvadorensis y anfípodos gamaridos. Los bivalvos representados por Donax dentifer estuvieron restringidos a las playas Dominical, Donimicalito, Violines y Venegas. Los nemertinos se presentaron con cantidad considerable en la región de Punta Burica. La densidad para la zona en general es de 736.9 individuos $/ \mathrm{m}^{2}$. La riqueza de taxones no mostró diferencia entre sitios $\left(\chi^{2}=13.5\right.$, d.f $=19, \mathrm{p}=0.834)$, las playas presentaron de 1 a 7 taxones como máximo. La abundancia total varió entre las playas $\left(\chi^{2}=399.91\right.$, d. $f=19, p<$
0.001), desde un individuo a 61, como se muestra en el Cuadro 1.

El análisis de conglomerados (Fig. 2), muestra que seis playas del Pacífico Sur presentan mucha diferencia en composición de infauna, al compararlas con el resto de playas estudiadas y entren sí mismas, debido a los parches característicos de especies presentes. Así, Punta Mangle difiere del resto de sitios, esto por poseer una gran abundancia de gusanos nereididos. Las playas Violines y Garza se apartan del resto por la abundancia de colémbolos que se encontraron en el sedimento de éstas. La playa Piñuelas, es la única playa que se distingue en el sector de Marino Ballena por la cantidad de anfípodos que presentó. Palo Seco y Venegas presentaron pocos colémbolos y el bivalvo $D$. dentifer en su infauna (Cuadro 1, Fig. 2). Estas playas por lo general fueron las que más abundancia total presentaron y todas son del tipo disipativas. Los sitios más al norte de Punta Burica (Punta Banco, Coco, Caña Blanca) y playa La Palma, tienden a formar un grupo, que coincide en la presencia de nemertinos que se adhieren a los granos de arena y el gusano Pisione remota. Estas son playas reflectivas o intermedias en general. Dominicalito y Uvita son playas donde la fauna más común fue el isópodo C. salvadorensis y gasterópodos de la familia Olividae. Dominicalito presentó una abundancia alta de este isópodo, siendo también una playa disipativa. Los otros sitios se agrupan como lugares de muy baja abundancia (Cuadro 1, Fig. 2), en varios casos, con playas más expuestas del tipo intermedio o reflectivas.

La distribución vertical de la infauna para la zona de Punta Burica, revela que se da un incremento en el número de taxones y en la abundancia total conforme se llega al nivel de la marea baja, mientras el supralitoral presenta solo dos taxones (Cuadro 2). Los grupos varían en su distribución, se encontraron 13 taxones en la zona infralitoral y litoral bajo, como el gusano glicérido y crustáceos. El gusano depredador nereidido y $P$. remota son dominantes en la zona baja de la playas de la región de Punta Burica, mostrando ambos una reducción hacia el supralitoral donde están 


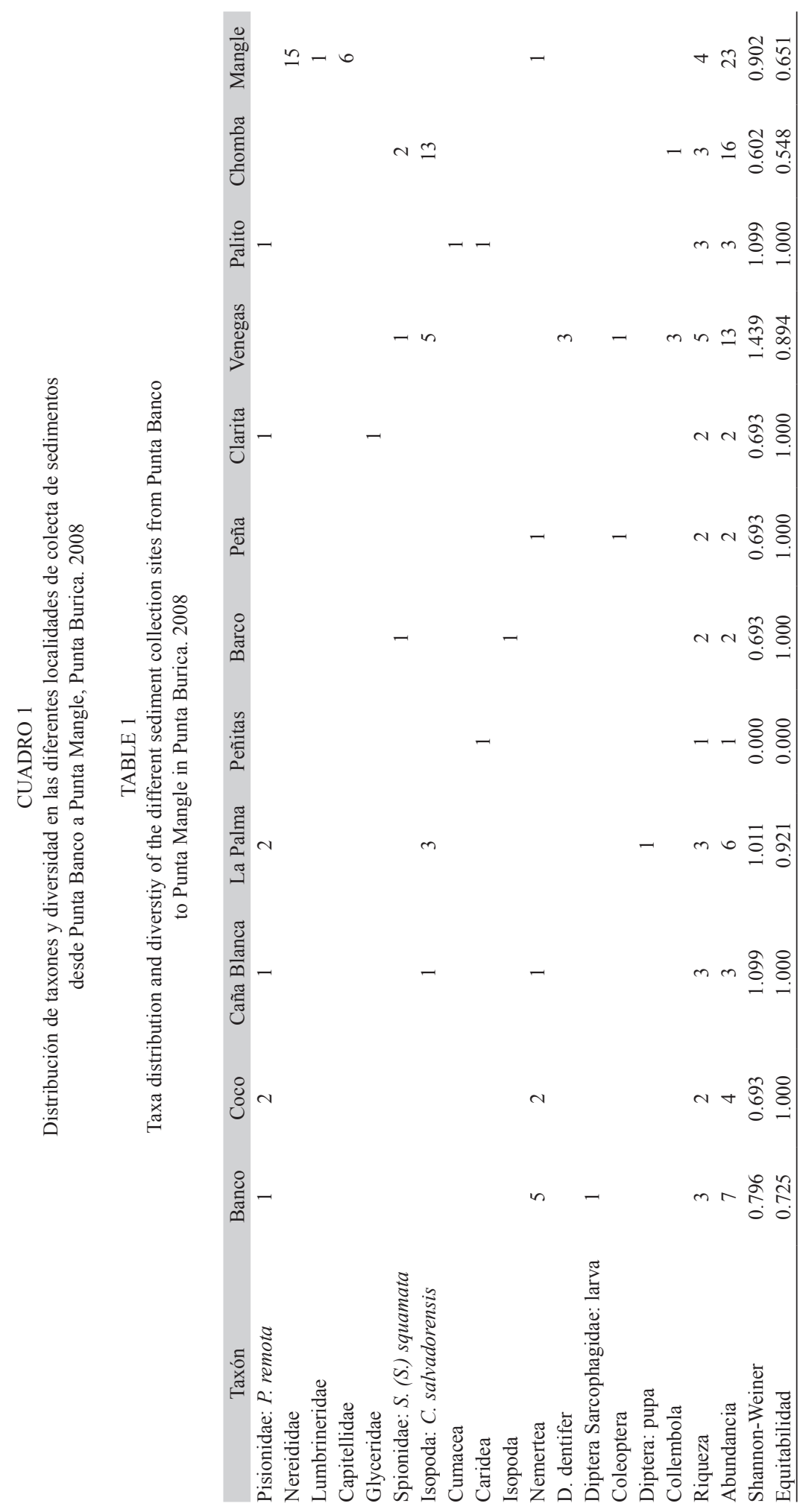




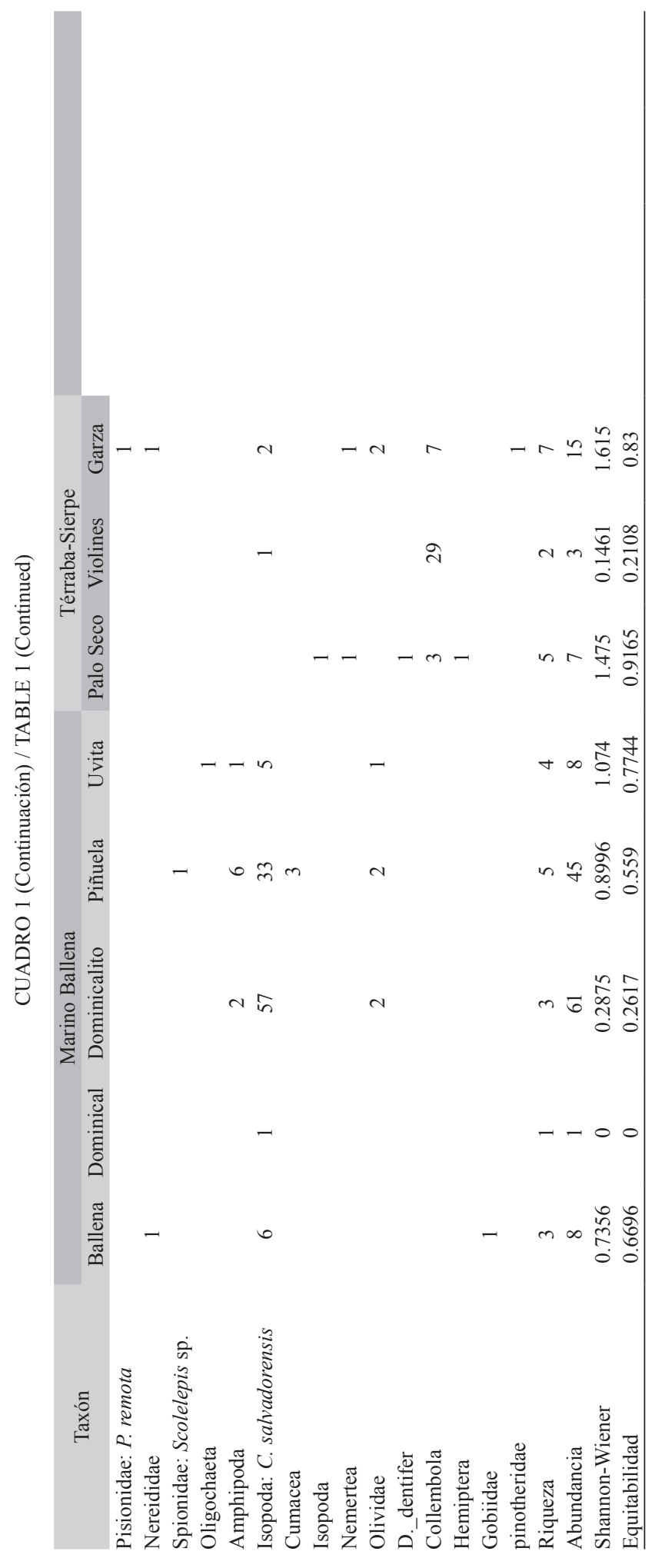




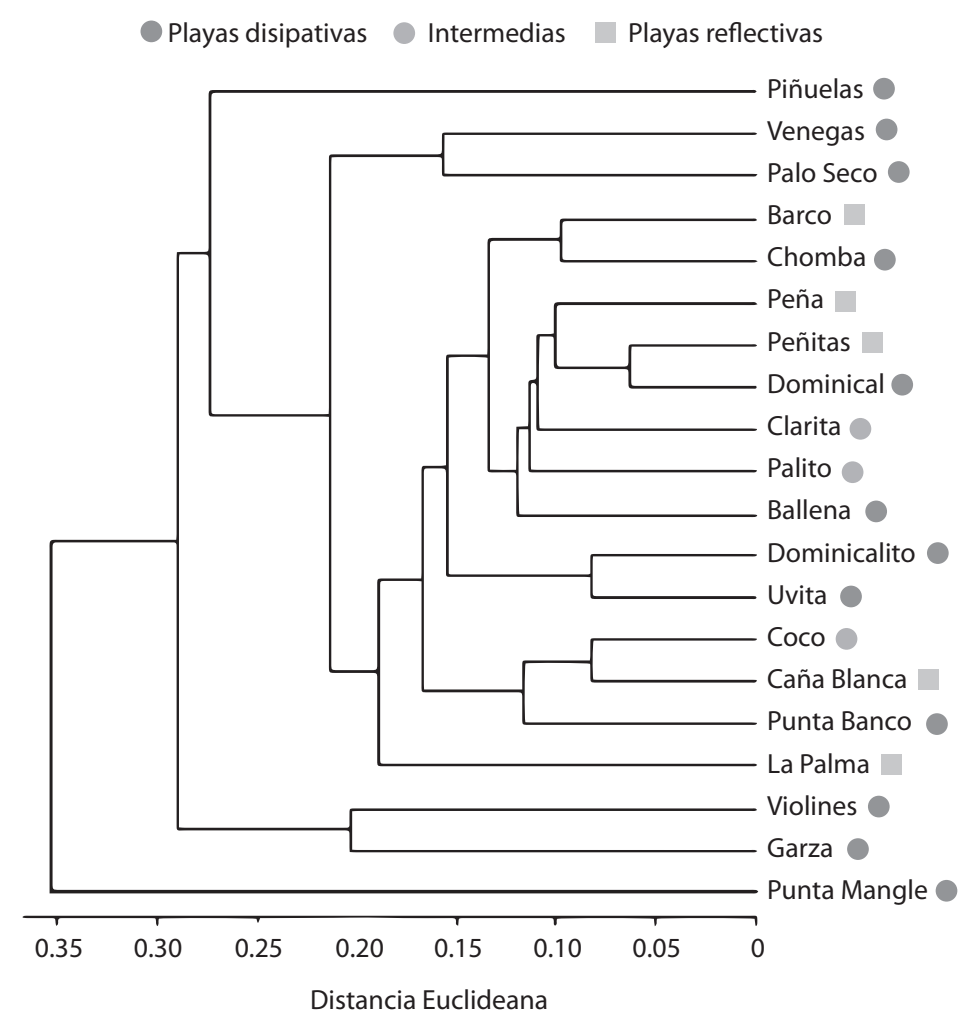

Fig. 2. Análisis de conglomerados mostrando la similitud de la fauna de las playas de arena, según su clasificación física, en las regiones de Marino Ballena, Térraba Sierpe y Punta Burica, Puntarenas, Costa Rica. 2008-2012.

Fig. 2. Cluster analysis that shows the similitude of the macrofauna composition found in sandy beaches according to physical classification. The beaches included are found on the Marino Ballena, Térraba Sierpe and Punta Burica regions, Puntarenas, Costa Rica. 2008-2012.

ausentes. Por otro lado los bivalvos estuvieron presentes a medio intermareal en la región de Punta Burica y el isópodo C. salvadorensis incrementa su abundancia hacia el litoral alto. Para los otros sitios estudiados, desde Dominical a Corcovado, se encontró un patrón similar. Por ejemplo, en la región de Dominical, se dieron varios taxones en el intermareal bajo, entre los cuales destacan crustáceos como los anfípodos, isópodos y cumáceos. En la región supralitoral, se encontraron también anfípodos y el isópodo C. salvadorensis.

Otros grupos que no fueron recolectados en los barrenos, pero que se avistaron en las playas (Cuadro 3 y 4), fueron el cangrejo ermitaño Coenobita compressus en Marino Ballena, Sierpe-Terraba y Corcovado, el cangrejo topo
(Emerita rathbunae) fue observado en todas las playas de la región de Marino Ballena y en playa Violines. En Punta Burica fue encontrado en la Peña y cerca de Caña Blanca siempre en el infralitoral. La galleta de mar (Mellita longifisa) fue encontrada en playa Uvita, Ballena y Piñuelas. En Punta Burica se encontró además el poliqueto Diopatra ornata en el playón de Roca Barco, con otros poliquetos, gastrópodos y jaibas de la especie Callinectes toxodes, las cuales se observaron también en dos playas de la región de Marino-Ballena y en todas las playas de Sierpe-Térraba (Cuadro 3). Estas especies, mayores en tamaño, también siguen una distribución vertical a lo largo de las playas visitadas, como se ejemplifica en el perfil para Punta Barco, en Burica (Cuadro 3, 
CUADRO 2

Zonación vertical de 12 playas de arena de la región entre Punta Banco y Punta Mangle, Punta Burica. 2008

TABLE 2

Vertical zonation of 12 sandy beaches between Punta Banco and Punta Mangle in Punta Burica. 2008

\begin{tabular}{|c|c|c|c|c|c|c|}
\hline & \multirow{2}{*}{ Taxón } & \multicolumn{5}{|c|}{ Zona } \\
\hline & & Infralitoral & Litoral bajo & Litoral medio & Litoral alto & Supralitoral \\
\hline Glyceridae & & 1 & & & & \\
\hline Isopoda & Isopoda & 1 & & & & \\
\hline Cumacea & & 1 & & & & \\
\hline Diptera & Sarcophagidae: larva & & 1 & & & \\
\hline Lumbrineridae & & & 1 & & & \\
\hline Malacostraca & Caridea & 1 & & 1 & & \\
\hline Pisionidae & P. remota & 3 & 3 & 2 & & \\
\hline Nereididae & & 8 & 2 & 4 & 1 & \\
\hline Nemertea & & 2 & 1 & 1 & 6 & \\
\hline Capitellidae & & 1 & 4 & 1 & & \\
\hline Collembola & & 2 & 1 & & 1 & \\
\hline Spionidae & $S$. (S.) squamata & & 2 & & 2 & \\
\hline Bivalvia & D. dentifer & & & 1 & 2 & \\
\hline Coleoptera & & & & 1 & 1 & \\
\hline Isopoda & C. salvadorensis & & 1 & 5 & 7 & 9 \\
\hline Diptera & Pupa & & & & & 1 \\
\hline Total de taxones & & 9 & 9 & 8 & 7 & 2 \\
\hline Abundancia total & & 20 & 16 & 16 & 20 & 10 \\
\hline
\end{tabular}

CUADRO 3

Organismos encontrados en el playón arenosos de playa Barco. 2008

TABLE 3

Organisms found in sandy beach of playa Barco, Punta Burica. 2008

\begin{tabular}{|c|c|c|}
\hline Filo & Familia & Genero \\
\hline \multirow[t]{4}{*}{ Annelida } & Onuphidae & Diopatra sp. \\
\hline & Orbiniidae & Scoloplos sp. \\
\hline & Glyceridae & \\
\hline & Magelonidae & Magelona sp. \\
\hline \multirow[t]{3}{*}{ Mollusca } & Donacidae & Donax dentifer \\
\hline & Olivelidae & Olivella semiestriata \\
\hline & Collumbelidae & Anachis sp. \\
\hline \multirow[t]{3}{*}{ Crustacea } & Grapsidae & Sesarma sp. \\
\hline & Squillidae & \\
\hline & Portunidae & Callinectes toxodes \\
\hline Echinodermata & Mellitidae & Mellita longifissa \\
\hline
\end{tabular}

4, Fig. 3). Finalmente, cangrejos de la familia Ocypodidae, principalmente de género $U c a$, fueron vistos en varias localidades como Punta
Mangle, Punta Barco y Coco. El género Ocypode fue observado en todas las playas de las otras tres regiones.

\section{DISCUSIÓN}

La cantidad de especies encontrada en este estudio es baja si se compara con otras playas de la costa pacífica costarricense. En Punta Morales, Golfo de Nicoya, una playa fangosa, protegida del oleaje fuerte, se reportan 93 especies (Vargas, 1987). Los valores siguen siendo bajos si se comparan con los 22 taxones de Playa Jaco, pero son similares a otras playas arenosas en ese mismo estudio (Dexter, 1974), como Sámara y Tamarindo (15), El Coco (13), Barranca y Cocal (Quepos) (16). El valor es alto para ciertas playas del Pacífico Sur. La densidad sobre los setecientos individuos $/ \mathrm{m}^{2}$ es intermedio a los valores reportados por Dexter (1974). El valor es bajo en relación con los presentados por Alongi (1989), para varias 
CUADRO 4

Presencia de Taxones en las áreas de estudio del Pacífico Sur de Costa Rica

TABLE 4

Taxa present in the different study areas of the Southern Pacific coast of Costa Rica

\begin{tabular}{|c|c|c|c|c|c|}
\hline & \multirow{2}{*}{ Taxones } & \multicolumn{4}{|c|}{ Áreas de estudio } \\
\hline & & Marino-Ballena & Térraba & Corcovado & Punta Burica \\
\hline \multirow[t]{9}{*}{ Crustacea } & Amphipoda & $\mathrm{X}$ & $\mathrm{X}$ & $\mathrm{X}$ & \\
\hline & Cirolana salvadorensis & $\mathrm{X}$ & $\mathrm{X}$ & $\mathrm{X}$ & $\mathrm{X}$ \\
\hline & Callinectes toxodes & $\mathrm{X}$ & $\mathrm{X}$ & & $\mathrm{X}$ \\
\hline & Caridea & & & & $\mathrm{X}$ \\
\hline & Cumacea & $\mathrm{X}$ & & & $\mathrm{X}$ \\
\hline & Ocypode sp. & $\mathrm{X}$ & $\mathrm{X}$ & $\mathrm{X}$ & \\
\hline & Uca sp. & & & & $\mathrm{X}$ \\
\hline & Coenobita compressus & $X$ & $\mathrm{X}$ & $\mathrm{X}$ & \\
\hline & Emerita rathbunae & & $\mathrm{X}$ & & $\mathrm{X}$ \\
\hline Echinodermata & Mellita longifissa & $\mathrm{X}$ & $\mathrm{X}$ & & $\mathrm{X}$ \\
\hline \multirow[t]{3}{*}{ Mollusca } & Olividae & $\mathrm{X}$ & $\mathrm{X}$ & & $\mathrm{X}$ \\
\hline & Natica sp. & & $\mathrm{X}$ & & \\
\hline & Donax. dentifer & $\mathrm{X}$ & $\mathrm{X}$ & & $\mathrm{X}$ \\
\hline \multicolumn{6}{|l|}{ Nemertea } \\
\hline \multirow[t]{7}{*}{ Polychaeta } & Nereidae & $\mathrm{X}$ & $\mathrm{X}$ & & $\mathrm{X}$ \\
\hline & Onuphidae & & $\mathrm{X}$ & & $\mathrm{X}$ \\
\hline & Scolelepis squamata & $\mathrm{X}$ & $\mathrm{X}$ & $\mathrm{X}$ & $\mathrm{X}$ \\
\hline & Pisionidae & & $X$ & & $X$ \\
\hline & Lumbrineridae & & & & $\mathrm{X}$ \\
\hline & Capitellidae & & & & $\mathrm{X}$ \\
\hline & Glyceridae & & & & $\mathrm{X}$ \\
\hline \multirow[t]{3}{*}{ Hexapoda } & Diptera & & & & X \\
\hline & Coleoptera & & & & $X$ \\
\hline & Collembola & & $\mathrm{X}$ & & $\mathrm{X}$ \\
\hline
\end{tabular}

playas de arena tropicales con más de dos mil individuos $/ \mathrm{m}^{2}$. Si se compara Punta Burica con playas de México (193ind $\left./ \mathrm{m}^{2}\right)$, muestra valores más altos la primera (Alongi, 1990).

En cuando a la composición de la fauna es similar a la reportada por Dexter (1974), con C. salvadorensis en el litoral alto y supralitoral. Dexter menciona como poliquetos comunes en algunas de las playas de arena del Pacífico de Costa Rica a $S$. squamata y P. remota, y bivalvos del genero Donax ocurren en ambas costas. Alongi (1990) reporta para playas de arena tropicales del mundo, grupos similares de organismos, como característicos de estos ambientes. La distribución vertical de estos grupos es impuesta por el gradiente de desecación (Vohra, 1971), donde las formas blandas sin concha o exoesqueleto ocurren en el litoral bajo e infralitoral. La distribución vertical vista por grupos es parecida a la encontrada en Chile por Brazeiro et al. (1998), con insectos coleópteros en el litoral alto, así como isópodos cirolánidos (litoral alto y medio), poliquetos Scolelepis y anomuros Emerita en el litoral bajo, como en algunas de las playas del presente estudio. Las 10 especies que componen el $88 \%$ de la fauna de playas arenosas analizadas por Dexter (1974) fueron encontradas en este estudio.

Eleftheriou y Nicholson (1975), mencionan que playas de alta dinámica (reflectivas) 


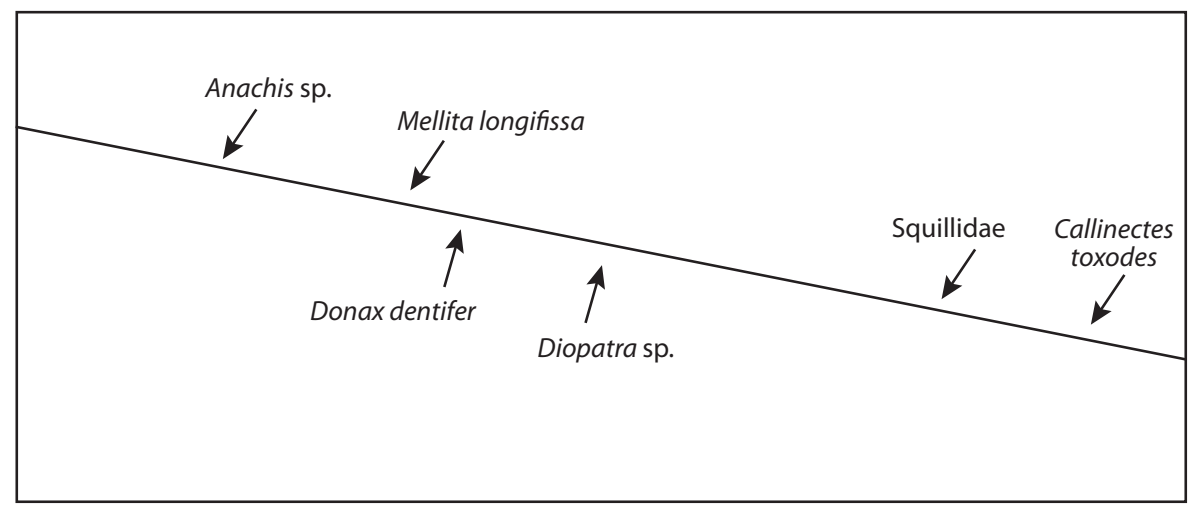

Fig. 3. Distribución vertical de los principales grupos de invertebrados encontrados en el playón arenoso de Playa Barco, desde el supralitoral (izquierda) hasta el infralitoral (derecha). Punta Burica, 2008.

Fig. 3. Vertical distribution of the main invertebrate taxa found Playa Barco, from the upper intertidar (left) to the lower intertidal (right). Punta Burica, 2008.

están dominadas por buenos nadadores como isópodos, playas de mediana exposición (intermedias) pueden presentarse bivalvos y poliquetos que forman túneles o galerías, así como algunos poliquetos errantes. Las playas de las regiones analizadas, presentan diferente exposición a la fuerza del oleaje que es la causa en parte de la variación en su fauna. Así, solo en algunas playas fue posible encontrar taxones sedentarios o gusanos que se alimenten del sedimento, mientras los organismos errantes y carnívoros, fueron la regla general.

Las playas reflectivas compuestas de arena con partículas más grandes, no presentan los estratos del litoral medio e infralitoral (McLachlan \& Jaramillo, 1995), lo cual se ve reflejado en el bajo número de especies (Brazeiro et al., 1998; Little, 2000), similar a lo encontrado en el presente estudio. Así, playas de la Península de Osa como San Pedrillo y la Chancha y la región de Punta Burica que se extiende desde El Coco hasta La Peña, mostraron solo una o dos especies; mientras, Venegas y Punta Mangle, de menor inclinación presentan más especies, siendo playas disipativas según los criterios de Little (2000).

McLachlan y Jaramillo (1995) determinaron que en aquellas playas disipativas que presentan cambios drásticos en la marea, el estrato infralitoral se subdivide en dos estratos: el primero incluye la zona efluente y el segundo delimitado por la línea de marea baja. Dichos variantes en la composición de la zonación ocurren en playas claramente disipativas como Violines y el playón de playa Barco, donde el sedimento es más fino, las playas tienen poca pendiente y están protegidas del oleaje extremo, en el caso de playa Barco por la Roca el Barco. Bajo estas condiciones se encontraron más poliquetos formadores de tubos como Magelona y Diopatra. Estas playas presentan una sección donde abundan las galletas de mar y otra donde se dan agregaciones del poliqueto Diopatra sp., similar al playón de Cocorocas del Golfo de Nicoya (Dittmann \& Vargas, 2001), pero con la variante de que en Violines y Burica la posición de las galletas de mar es en el litoral medio y no en el infralitoral. En Cocorocas, Golfo de Nicoya, la especie más común de galleta de mar es Encope stokesi, de menor talla y posiblemente se vea más afectado por la desecación que $M$. longuifissa. El gastrópodo Natica $\mathrm{sp}$., característico de playas con sedimento fino y que ha sido reportado en el Golfo de Nicoya (Maurer et al., 1984) sólo fue encontrado en la región infralitoral de Violines. Las características físicas de dicha playa están influenciadas por la descarga de sedimentos del delta estuarino Térraba-Sierpe, que en el Pacífico costarricense es considerable (Denyer Cárdenes, 2000). Además, la región presenta una importante corriente costera (Denyer \& 
Cárdenes, 2000), las cuales movilizan el sedimento (Little, 2000). Estos factores pueden provocar que el sedimento de Playa Violines tenga cierta cantidad de materia orgánica y por lo tanto presenta organismos que no se observan en otros ambientes intermareales cercanos.

Amenazas: Pese a la baja diversidad encontrada en playas arenosas con relación a otros ecosistemas marinos, la macrofauna de las playas de arena es característica y única, por lo cual de debe ser protegida. Una amenaza constante para el hábitat entre mareas es su utilización como vía de comunicación en los sectores al sur del Río Peña hasta Punta Mangle. En este trayecto se da el tránsito de vehículos gracias a la amplitud de la zona expuesta durante la marea baja. Schlacher y Thompson (2007), en Australia mencionan el traslape de la mayor parte de la comunidad de entre mareas con el tránsito de vehículos por la playa y como éstos afectan en la distribución de la abundancia de la especies. En muchos casos se dan disturbios sobre la estabilidad del sedimento así como compactación. Van der Merwe y Van der Merwe (1991), concluyen que el aumento en tránsito incrementa los daños en las especies menos tolerantes como isópodos del supralitoral donde la arena es menos compacta. Roy (2007) señala que el pisoteo puede afectar a pequeños invertebrados que son aplastados. Para la región desde Punta Banco hasta Caña Blanca, los disturbios son producidos por el uso de caballos como transporte.

El turismo en la región de Punta Burica se encuentra en niveles bajos, lo cual contrasta con la alta visitación por turistas a las playas desde Dominical hasta Marino-Ballena, lo cual genera un efecto negativo sobre la fauna en el sedimento, por disturbios en el mismo o extracción de organismos para colección y alimento, ambos factores producen una disminución de las poblaciones naturales de invertebrados en zonas intermareales (Roy, 2007).

También, se observó acumulación de basura a lo largo de la costa, desde botellas plásticas hasta llantas, tanques de gas y material sanitario, transportadas por corrientes desde los centros de mayor población. Otros impactos obvios en las playas más al norte, cerca de Marino Ballena y potenciales en Sierpe, Corcovado y Punta Burica, son movimientos masivos de tierra durante construcción en las zonas aledañas, incluida la extracción de arena de las playas para construcción a gran escala. Los impactos antropogénicos pueden ser la causa de la baja diversidad encontrada en playas disipativas de las UEM Ballena y Burica. El análisis de conglomerados agrupa estas playas junto con las reflectivas, las cuales se espera que presenten menor número de especies debido a su dinámica (McLachlan \& Jaramillo, 1995; Little, 2000).

\section{CONCLUSIONES}

Las zonas arenosas de las regiones estudiadas presentan más bien una diversidad intermedia si se compara con otras playas de arena, con excepción de las playas de Corcovado, que presentan una diversidad baja. El patrón principal es que cuanto más reflectiva la playa menos cantidad de especies la habitan. Playa Violines y el playón del Barco son variantes en la región, pues contienen una fauna diferente a las playas de arena, al estar más protegidas del oleaje fuerte y en el caso de Violines, presentar un sedimento con mayor cantidad de materia orgánica. En ambos sitios, las especies son similares a las reportadas previamente en otras playas del país. La baja diversidad en las playas del Pacífico Sur puede deberse a impactos humanos que se dan en las zonas de diversa manera e intensidad.

\section{AGRADECIMENTOS}

Se agradece a Fundación Keto y TNC por brindar el financiamiento y apoyo logístico para realizar este trabajo. Se agradece a los COVIRENAS Sebastián Rodríguez Santos y Javier Guerra Montezuma por su ayuda en el trabajo de campo. A Silvia Solano y a Fundación Keto por facilitar los estereoscopios. Al Proyecto Consolidación de Áreas Marinas 
Protegidas del Sistema Nacional de Áreas de Conservación (SINAC), Programa de Naciones Unidas para el Desarrollo (PNUD) y el Global Environment Facility (GEF) por su colaboración en la publicación y al proyecto GIZ.

\section{RESUMEN}

Se describe la diversidad y composición de especies las playas de arena del Pacífico Sur de Costa Rica. En las playas de arena de la región, se colectó individuos de la infauna por medio de barrenos. Para la zona de entre mareas arenosa el número de especies estrictamente marina varió de 5 a 13 taxones, número que es intermedio con los reportados previamente para estos ambientes en Costa Rica. En la zonas de arena, el supralitoral lo dominó el isópodo Cirolana salvadorensis, mientras nereidos y un gusano de la Familia Pisionidae dominaron en el infralitoral. Otros organismos encontrados en las playas fueron cangrejos del género Uca, cangrejos anomuros (Emerita), galletas de mar (Mellita longifissa) y varios grupos de poliquetos tubícolas permanentes o temporales de la familias Onuphidae, Spionidae, Magelonidae y Glyceridae. Las playas mostraron variación en su fauna atribuible en ciertos casos a la exposición al oleaje, teniendo menos fauna en las playas más reflectivas. Se mencionan acitivades humanas que pueden explicar la baja riqueza de especies encontrada en algunas de las playas analizadas.

Palabras clave: Playa de arena, zonación vertical, biodiversidad marina, Punta Burica, impactos humanos.

\section{REFERENCIAS}

Alongi, D. M. (1989). Ecology of tropical soft-bottom benthos: a review with emphasis on emerging concepts. Revista de Biología Tropical, 37, 85-100.

Alongi, D. M. (1990). The Ecology of Tropical SoftBottom Benthic Ecosystems. Oceanography and Marine Biology: an Annual Review, 28, 381-496.

Brazeiro, A., Rozbaczylo, N., \& Fariña, J. M. (1998). Distribución espacial de la macrofuana en una playa expuesta de Chile central: efectos de la morfología intermareal. Investigaciones Marinas, 26, 119-126.

Cortés, J., \& Jiménez, C. E. (1996). Coastal marine environments of Parque Nacional Corcovado, Puntarenas, Costa Rica. Revista de Biología Tropical, 44(3), $35-40$.

Dean, H. K. (2004). Marine biodiversity of Costa Rica: Class Polychaeta (Annelida). Revista de Biología Tropical, 52(2), 131-181.

De la Cruz, E., \& Vargas, J. A. (1987). Abundancia y distribución vertical de la meiofauna en la playa fangosa de Punta Morales, Golfo de Nicoya, Costa Rica. Revista de Biología Tropical, 35, 363-367.

Denyer, P., \& Cárdenes, G. (2000). Costas Marinas. In P. Denyer \& S. Kussmaul (Eds.), Geología de Costa Rica (pp 185-218). Cartago, Costa Rica: Editorial Tecnológica de Costa Rica.

Dexter, D. M. (1974). Sandy beach fauna of the Pacific and Atlantic coast of Costa Rica and Colombia. Revista de Biología Tropical, 22, 51-66.

Dittmann, S. (2000). Zonation of benthic communities in a tropical tidal flat of north-east Australia. Journal of Sea Research, 43, 33-51.

Dittmann, S., \& Vargas, J. A. (2001). Tropical tidal flat benthos compared between Australia and Central America. In K. Reise (Ed.), Ecological Comparisons of Sedimentary Shores. Ecological Studies (pp. 275-293). New York, USA.; Springer-Verlag, Berlin, Heidelberg.

Eleftheriou, A., \& Nicholson, M. D. (1975). The effects of exposure on beach fauna. Cahiers de Biologie Marine, 16, 695-710.

Giere, O. (2008). Meiobenthology: The Microscopic Motile Fauna of Aquatic Sediments. Berlin, Germany: Springer-Verlag Berlin Heidelberg.

Gray, J. S., \& Elliot, M. (2009). Ecology of Marine Sediments, from Science to Management. New York, USA.: Oxford University Press Inc.

Hammer, Ø., Harper, D. A. T., \& Ryan, P. D. (2001). Past: Paleontological Statistics Software Package for Education and Data Analysis. Palaeontologia Electronica, 4, 1-9.

Høisæter, T. (1998). Preliminary check-list of the marine, shelled gastropods (Mollusca) of Golfo Dulce, on the Pacific coast of Costa Rica. Revista de Biología Tropical, 46(6), 263-270.

Little, C. (2000). The Biology of Soft Shores and Estuaries. Oxford: Oxford Univ. Press.

McLachlan, A., \& Jaramillo, E. (1995). Zonation on sandy beaches. Oceanography and Marine Biology: An Annual Review, 33, 305-355.

Maurer, D., Epifanio, C., Dean, H., Howe, S., Vargas, J., Dittel, A., \& Murillo, M.. (1984). Benthic invertebrates of a tropical estuary: Gulf of Nicoya, Costa Rica. Journal of Natural History, 18, 47-61.

Quesada-Alpízar, M., \& Cortés, J. (2006). Los ecosistemas Marinos del Pacífico Sur de Costa Rica: estado del conocimiento y perspectivas de manejo. Revista de Biología Tropical, 54(1), 101-145.

Roy, K. (2007). Ecosystem Changes, Natural versus Anthropogenic. In M. Denny \& S. Gaines (Eds.), Encyclopedia of Tidepools and Rocky Shores (pp. 201-204). London, England: University of California Press Ltd. 
Schlacher, T. A., \& Thompson, L. M. C. (2007). Exposure of fauna to off-road vehicle (ORV) traffic on sandy beaches. Coastal Management, 35, 567-583.

Sistema Nacional de Áreas de Conservación (SINAC). (2009). Grúas II. Propuesta de ordenamiento territorial para la conservación de la biodiversidad de Costa Rica. Análisis de vacios de conservación en Costa Rica. Vol III (1ra ed.). San José, Costa Rica: Asociación Conservación de la Naturaleza.

Van der Merwe, D., \& Van der Merwe, D. (1991). Effects of off-road vehicles on the macrofauna of a sandy beach. South African Journal of Science, 87, 210-213.
Vargas, J. A. (1987). The benthic community of an intertidal mud flat in the Gulf of Nicoya, Costa Rica. Description of the community. Revista de Biología Tropical, 35, 229-316.

Vargas, J. A. (1988a). Community structure of macrobenthos and the results of macrodepredator exclusion on a tropical mud flat. Revista de Biología Tropical, 36, 287-308.

Vargas, J. A. (1988b). A survey of the meiofauna of an eastern tropical Pacific intertidal mud flat. Revista de Biología Tropical, 36, 541-544.

Vohra, F. C. (1971). Zonation on a tropical sandy shore. Journal of Animal Ecology, 40, 679-708. 
